\title{
Modelo de autoevaluación integrado entre la norma ISO 9004 y el sistema único de acreditación en salud ${ }^{*}$
}

\section{Self-assessment model integrated between ISO 9004 and the Sistema Único de Acreditación en Salud}

Yolvi Amileth Rodrígues Navas"

Juan Pablo Robayo Piñeros"

Convenio Universidad Santo Tomás e ICONTEC

\section{RESUMEN}

Todo proceso de mejora permanente en las organizaciones requiere de una reflexión periódica sobre el camino recorrido, los avances, los tropiezos y los retos que se presentan, para el logro de los propósitos organizacionales. La autoevaluación, como proceso interno de valoración, se constituye en una herramienta valiosa para llevar a cabo dicha reflexión.

El modelo de gestión de la calidad ISO 9004:2009 contiene una herramienta para llevar a cabo una autoevaluación, la cual permite identificar oportunidades para la mejora, mostrar fortalezas y debilidades para lograr el éxito sostenido, establecer el nivel de madurez de

* Artículo de investigación.

** Docente del Programa de Fisioterapia en la Universidad de Boyacá. Fisioterapeuta, Especialista en Gestión en Rehabilitación. Correo electrónico: yolviamilethrodriguez@gmail.com

***Fundación Hospital San Carlos. Médico, Especialista en Salud Ocupacional. Correo electrónico: juanprobayop@gmail.com

Trabajo de grado para optar al título de Magíster en Calidad y Gestión Integral, dirigido por: Mg. José Edilberto Pinzón Quiroga. 
la organización y, si se repite, determinar el progreso de la organización en el tiempo. Dado el enfoque generalista de la norma ISO 9004, esta herramienta es aplicable a cualquier tipo de organizaciones.

Por otra parte, el sistema único de acreditación para las instituciones de salud en Colombia incluye también una herramienta de autoevaluación con una especificidad muy alta para el sector salud, pero que no tiene el enfoque sistémico de ISO 9004: no permite determinar un nivel de madurez y, según los usuarios, resulta complejo de aplicar.

Se percibe entonces la posibilidad de crear un instrumento metodológico que integre lo mejor de ambas herramientas y resulte práctico en su aplicación.

Este documento presenta los resultados de la aplicación de la herramienta de autoevaluación del modelo ISO 9004:2009 en una institución prestadora de servicios de salud de alto nivel de complejidad y su comparación con el resultado de la autoevaluación del sistema único de acreditación.

Este ejercicio permitió identificar las similitudes, diferencias, fortalezas y debilidades de los dos instrumentos, lo cual condujo a la vez, dentro de una orientación de complementariedad e integración de modelos de gestión de la calidad, a desarrollar y presentar una nueva propuesta de instrumento de autoevaluación, enriquecido y fortalecido, aplicable a cualquier institución de salud que desee optar por la acreditación.

Palabras clave: autoevaluación, nivel de madurez, Norma ISO 9004:2009, sistema único de acreditación en salud, sistema de gestión de la calidad.

\section{ABSTRACT}

Any process of continuous improvement in organizations requires a periodic reflection regarding the path, progress, setbacks and challenges that arise while striving to achieve organizational purposes. Selfassessment, as an internal assessment process, constitutes a valuable tool for conducting such a reflection.

The ISO 9004:2009 model of quality management contains a tool to conduct a self-assessment, which identifies opportunities for improvement, shows strengths and weaknesses to achieve sustained success, establishes the level of maturity of the organization and, if repeated, determines the progress of the organization over time; given the general approach of ISO 9004, this tool is applicable to any type of organization.

Moreover the Sistema Único de Acreditación for health institutions in Colombia, also includes a self-assessment tool with very high specificity for the health sector, but this does not have the systemic approach of ISO 9004, it is not possible to determine a level of maturity and, according to users, it is complex to implement.

The possibility is then perceived of creating a methodological tool that integrates the best of both tools and is practical in application.

This paper presents the results of applying the ISO 9004: 2009 model assessment tool in a high level of complexity health servicing institution and its comparison with the result of the self-assessment of the Sistema Único de Acreditación.

This exercise identified the similarities, differences, strengths and weaknesses of the two instruments, which led in turn to a complementary orientation and integration of models of quality management, to develop and 
present a new proposal for an instrument of self-evaluation, enriched and strengthened, applicable to any health institution which wants to opt for accreditation.

Keywords: self-assessment, maturity level, ISO 9004:2009, quality management system.

\section{INTRODUCCIÓN}

Prestar servicios de salud dentro de los mejores estándares de calidad debe ser el propósito de toda organización y/o profesional que labore en este campo, razón por la cual todo mecanismo o estrategia que se use y se disponga para el logro de tal fin resulta de gran utilidad en la búsqueda del cumplimiento de este objetivo; de ahí la importancia de vislumbrar alternativas de monitoreo y medición de los niveles de desarrollo de empresas de salud mediante procesos de autoevaluación que permitan que una institución esté siempre en el sendero del éxito sostenido.

La búsqueda permanente de la excelencia en sectores empresariales que trabajan en la gestión para el éxito sostenido ha llevado a implementar la NTCISO 9004, proceso que implica realizar una revisión exhaustiva y sistemática de las actividades de la organización y su desempeño en relación con su grado de madurez en la gestión organizacional, a lo que se le denomina "autoevaluación". La NTC-ISO 9004, dentro de sus anexos, proporciona una herramienta de autoevaluación que tiene dos componentes: la autoevaluación de elementos clave y la autoevaluación de elementos detallados, los cuales incluyen elementos de: gestión, estrategia y política, recursos, procesos, seguimiento y medición y, por último, mejora, innovación y aprendizaje.

1 NTC - ISO 9004: 2009. Gestión para el éxito sostenido de una organización. Enfoque de Gestión de la Calidad.
Esta autoevaluación contiene cuarenta elementos que permiten evaluar el nivel de madurez en la gestión organizacional de una empresa, independientemente del tamaño, el tipo o la actividad, entendiendo que la madurez en la gestión organizacional indica que la organización está perfectamente condicionada para emprender todos sus proyectos de innovación, sean estos tecnológicos o de gestión integral de los recursos (Espinosa, Díaz y Back, 2008)².

En Colombia, el Ministerio de la Protección Social diseñó un sistema de gestión de la calidad para la salud (sistema obligatorio de garantía de la calidad) que está conformado estructuralmente por cuatro mecanismos de gestión ${ }^{3}$ : el sistema único de habilitación, la auditoría para el mejoramiento de la calidad de la atención de salud (PAMEC), el sistema único de acreditación y el sistema de información para la calidad; de estos componentes el único que no es de obligatorio cumplimiento (por lo menos sobre la normatividad legal vigente a la fecha, no así desde la perspectiva del marketing, en donde se hace imperativo competir por calidad para permanecer en los mercados) es el sistema único de acreditación.

El sistema único de habilitación (SUH) garantiza con sus requisitos las condiciones mínimas en las cuales debe funcionar una institución prestadora de salud, siendo el sistema único de acreditación (SUA) un modelo de excelencia y una herramienta de mejoramiento continuo que

2 ESPINOSA, Fernando F., DÍAZ, Acires y BACK, Nelson. Un procedimiento de evaluación de las condiciones necesarias para innovar la gestión de mantenimiento en una empresa. (spanish). En: Información Tecnológica. Vol. 19, No. 1 (enero, 2008): pp. 97103. Academic Search Complete, EBSCO host (consultado 16 de noviembre, 2010). Disponible en http://web.ebscohost.com/ehost/ detail? vid=3\&hid=104\&sid $=477761 b 1-7593-41 \mathrm{ad}-\mathrm{b} 8 \mathrm{dc}-00 \mathrm{e} 5 \mathrm{~b} 4 \mathrm{ef}$ 682f\%40sessionmgr112\&bdata=Jmxhbmc9ZXMmc210ZT1laG9zd $\mathrm{C} 1 \mathrm{saXZl} \# \mathrm{db}=\mathrm{a} 9 \mathrm{~h} \& \mathrm{AN}=31464154$

3 COLOMBIA. MINISTERIO DE SALUD Y PROTECCIÓN SOCIAL. Guía práctica del sistema obligatorio de garantía de la calidad en salud. Bogotá, D.C.: Ministerio de Salud y Protección Social, marzo de 2011.98 p. 
se convierte en el principal instrumento para garantizar a los usuarios los mejores estándares de calidad en la prestación de los servicios. Para alcanzar la acreditación, la organización debe examinar la operación de su gestión comparando el quehacer diario (calidad observada) versus los estándares y criterios del SUA (calidad deseada); a este ejercicio es a lo que se le llama "autoevaluación".

Dentro del SUA, cada institución debe autoevaluarse en diferentes momentos: durante el ciclo de preparación para la aplicación frente al ente acreditador, en la implementación de la ruta crítica de acreditación y en la identificación de procesos prioritarios objeto de mejoramiento ${ }^{4}$.

Es importante aclarar que para realizar la autoevaluación dentro del SUA, en la actualidad la institución prestadora de servicios de salud (IPS) que oferte diferentes servicios de salud deberá comparar sus procesos con los estándares del anexo técnico de la Resolución 123 de 2012 (Manual de acreditación en salud ambulatorio y hospitalario). Este manual mejoró y actualizó los estándares contenidos en el anexo técnico 1 de la Resolución 1445 de 2006, cuyo bloque de estándares se organiza de la siguiente manera: estándares del proceso de atención al cliente asistencial, estándares de direccionamiento, estándares de gerencia, estándares de gerencia del talento humano, estándares de gerencia del ambiente físico, estándares de gestión de la tecnología, estándares de gerencia de la información y estándar de mejoramiento de la calidad.

La autoevaluación del sistema único de acreditación (SUA) es una actividad en donde el cumplimiento de cada estándar y criterio se debe evaluar dentro del marco de las dimensiones del enfoque, la implementación y el resultado. Es un proceso dispendioso toda vez que para establecer el nivel de madurez de la gestión de la

4 Ibíd., p. 42. institución se deben verificar los más de 700 criterios que contiene la autoevaluación, además de la poca claridad en que están redactados cada uno de ellos, lo que da cabida a interpretaciones disímiles (dificultad que persiste aunque en menor proporción, a pesar de las mejoras de redacción del nuevo Manual de acreditación en salud ambulatorio y hospitalario normado a través de la Resolución 123 de 2012). Tales circunstancias generan incertidumbre, falta de interés y desmotivación en las IPS para el desarrollo e implementación del sistema.

La reflexión anterior se respalda en que actualmente en Colombia existen habilitadas 1144 IPS, de las cuales 969 son de nivel de complejidad 1 (84,70\%), 143 de complejidad $2(12,50 \%)$ y 32 de complejidad 3 $(2,80 \%)^{5}$, con tan solo 25 instituciones acreditadas ${ }^{6}$ en todo el país (2,1\% del total de IPS).

En ese orden de ideas, el escenario actual de Colombia, en relación con el monitoreo de la gestión con el enfoque en calidad de las IPS, es el de contar, primero, con el modelo de autoevaluación del SUA, que es específico para el sector salud, pero que es extenso, dispendioso, de no fácil interpretación, poco amable para diligenciar, con criterios no bien definidos de acuerdo con el logro del éxito sostenido, y segundo con el modelo de autoevaluación de la NTC ISO 9004 que, a pesar de ser de fácil aplicación, de poderse usar en cualquier organización según lo refiere esta norma internacional (aunque no existe conocimiento en Colombia de su utilización en el sector hospitalario),

5 COLOMBIA. MINISTERIO DE SALUD Y PROTECCIÓN SOCIAL. Prestación de servicios de salud. (2012). [consultado 5 de julio, 2012]. Disponible en http://201.234.78.38/SIHO/informes/ipspornivel.aspx?pageTitle=IPS\%20por $\% 20 \mathrm{Nivel} \% 20$ de\%20Atenci\%F3n\&pageHlp=/SIHO/ayudas/informes/caracterizacion.pdf

6 ICONTEC. Acreditación en salud. (2012). [consultado 5 de julio, 2012]. Disponible en http://www.acreditacionensalud.org.co/catalogo/docs/IPSacreditadas.pdf 
tiene el inconveniente de no contener elementos específicos para el sector de la salud.

En síntesis, el panorama de instituciones que prestan servicios de salud en el país es el de contar con la herramienta de autoevaluación del SUA, que no tiene un enfoque suficientemente global e integral para el logro del éxito sostenido, pero que es específica para el sector, y el de hacer uso de la matriz de autoevaluación de la norma ISO 9004:2009, que si bien orienta a las organizaciones en general hacia la consecución del éxito sostenido, no goza de elementos explícitos propios del sector sanitario. Al parecer, es por dicha razón que no ha sido utilizada en este sector.

Por lo anteriormente argumentado se propone una herramienta de autoevaluación del nivel de madurez organizacional en una IPS, que integre elementos y características de la NTC ISO 9004:2008 y el sistema único de acreditación en salud. La propuesta se surte técnica y metodológicamente de aplicar en una institución prestadora de servicios de salud de alta complejidad la autoevaluación de los elementos clave y detallados mediante el instrumento de autoevaluación del modelo ISO 9004:2009, a continuación se aplica la autoevaluación con el modelo del sistema único de acreditación en salud para identificar las relaciones entre los dos modelos de autoevaluación, para posteriormente diseñar un modelo que integre los elementos de la autoevaluación del modelo ISO 9004:2008 y el modelo de autoevaluación del sistema único de acreditación en salud.

\section{METODOLOGÍA}

Este trabajo hace parte de la línea de investigación Gestión de la Calidad de la Maestría en Calidad y Gestión Integral del Convenio USTA - ICONTEC. El enfoque es descriptivo, toda vez que contempla la descripción, registro, análisis e interpretación de la naturaleza actual, así como composición y/o procesos de los fenómenos; se hace sobre conclusiones dominantes o sobre cómo una persona, grupo o cosa se conduce 0 funciona en el presente ${ }^{7}$. El diseño es de tipo evaluativo, cuya meta, a diferencia de la investigación básica, no es el descubrimiento del conocimiento; proporciona información para la planificación del programa, su realización y su desarrollo. La investigación evaluativa asume también las particulares características de la investigación aplicada, que permite que las predicciones se conviertan en un resultado de la investigación?.

La metodología utilizada para recolectar información en la presente investigación se estructura en dos partes: una primera en donde se efectúa una revisión general de la literatura sobre el tema de estudio, y una segunda donde se realiza el trabajo de campo.

La primera parte, es decir la revisión general de la literatura sobre el tema de estudio, se realiza con el fin de identificar y analizar críticamente los elementos de la gestión de la calidad en salud, la aplicación de instrumentos de autoevaluación que determinen el nivel de madurez de una organización de salud en el sistema único de acreditación (SUA) y el modelo de autoevaluación de la norma ISO 9004, los cuales han sido validados por el Ministerio de Salud y Protección Social y la Organización Internacional de Normalización, respectivamente. Es decir, se identifican a nivel teórico elementos que permitan realizar la evaluación del nivel de madurez de una institución prestadora de servicios de salud de alto nivel de complejidad.

Posteriormente, se toma como referencia el instrumento de la NTC ISO 9004:2009 y se ajusta con otra columna donde se registran las evidencias que soportan

7 TAMAYO, Mario. El proceso de la investigación científica. México: Ed. Limusa, 2003. p. 46.

8 Ibíd., p. 25. 
el nivel de madurez seleccionado por las personas que llevarán a cabo el proceso de autoevaluación. De esta manera se aplica dicho instrumento, que tiene como objetivo evaluar el nivel de madurez de la gestión organizacional de una IPS de alto nivel de complejidad seleccionada por conveniencia.

La segunda parte, correspondiente al trabajo de campo, inicia con la aplicación del instrumento de la NTCISO 9004 de la siguiente manera: la autoevaluación de elementos clave, que consta de nueve preguntas, fue diligenciada por el delegado de la alta gerencia, y la autoevaluación de elementos detallados, que consta de 27 preguntas, por los profesionales del área de aseguramiento de la calidad. A continuación se solicitó información correspondiente a los resultados del proceso de autoevaluación con los estándares de los diferentes manuales de acreditación que aplican a la IPS. Luego de conocer a fondo cada modelo de autoevaluación, se diseñó un modelo integrador cuyo contenido fue validado por expertos.

Para la evaluación por expertos se escogió un grupo de tres evaluadores que analizaron la validez de contenido de los criterios seleccionados. El grupo que se eligió intencionalmente estuvo compuesto por expertos tanto en materia de acreditación en salud como en temas relacionados con investigación. De los tres evaluadores, dos son expertos en gestión de la calidad en salud y educación y el otro en investigación en educación superior.

Se remitió el instrumento al grupo de expertos seleccionados, con un instructivo que solicitaba valorar en una escala de cinco niveles el grado de congruencia, amplitud de contenido, redacción de los ítems, claridad y precisión de los distintos criterios planteados, donde $\mathrm{E}=$ excelente $(1) / \mathrm{B}=$ bueno $(0) / \mathrm{M}=$ mejorar $(-1) / \mathrm{C}=$ cambiar $(-1) / \mathrm{X}=$ Eliminar $(-1)$.

Finalmente, se procedió a la selección de los ítems con puntuación > 0,5 en el índice de Osterlind ${ }^{9}$ (1989) para formar parte del instrumento propuesto, lo que implica que más de la mitad de los expertos valoraron el ítem con un alto grado de idoneidad.

\section{RESULTADOS}

Se determinó el nivel de madurez organizacional de la IPS objeto de estudio a través del instrumento de la NTC ISO 9004; se tuvieron en cuenta procesos, procedimientos y documentos que en la actualidad hacen parte de los sistemas de gestión de dicha organización, y que aportan evidencia en cada uno de los siguientes elementos: 1. gestión; 2 . estrategia y política; 3 . recursos; 4. procesos; 5. seguimiento y medición; y 6. mejora, innovación y aprendizaje. Esta evaluación reportó un nivel 3 de madurez organizacional con un mínimo de 2 y un máximo de 4 en los valores de los elementos críticos y los elementos detallados, evidenciado en la siguiente tabla.

Tabla 1. Autoevaluación con instrumento de la NTC ISO 9004:2008

\begin{tabular}{|l|c|c|}
\hline Elementos & Clave & Detallados \\
\hline 1. Gestión & 2,5 & 3,3 \\
\hline 2. Estrategia y política & 3,0 & 3,3 \\
\hline 3. Recursos & 4,0 & 2,8 \\
\hline 4. Procesos & 3,0 & 2,5 \\
\hline 5. Seguimiento y medición & 2,5 & 3,3 \\
\hline 6. Mejora, innovación y aprendizaje & 3,0 & 3,3 \\
\hline & 3,0 & 3,1 \\
\hline
\end{tabular}

Fuente: Los autores, 2012.

9 MORALES, Pedro. Las pruebas objetivas. España: Editorial Universidad de Deusto, 2009. p. 60. 
El sistema único de acreditación (SUA) presenta los estándares que debe cumplir cada institución prestadora de servicios de salud, que para el caso de una institución de alto nivel de complejidad debe incluir los estándares de los manuales de acreditación de los servicios: laboratorio clínico, imagenología, habilitaciónrehabilitación y servicios hospitalarios-ambulatorios.

Para efectos prácticos en la autoevaluación de la IPS objeto de estudio, se elaboró una lista de chequeo en formato Excel que incluye cada uno de los estándares a los que debe dar cumplimiento.
Los resultados de la autoevaluación se presentan por grupo de estándares, tomando como medidas la media aritmética y la moda y empleando los criterios del SUA. Esta medición reporta como resultado un nivel de madurez de 2,6 por promedio y de 2 obtenido de la moda (téngase en cuenta que el sistema recomienda calificar usando como base el menor valor en la autoevaluación de los criterios), con puntajes que fluctúan con un valor mínimo de 2,3 y uno máximo de 2,9.

Tabla 2. Resultados de autoevaluación con el modelo SUA

\begin{tabular}{|l|c|c|}
\hline Grupo de estándares & \multirow{2}{*}{ Media } & Moda \\
\cline { 1 - 2 } Atención al cliente asistencial & 2,3 & 2 \\
\cline { 1 - 2 } Ambulatorios/hospitalarios & 2,8 & 3 \\
\hline Laboratorio clínico & 2,9 & 3 \\
\hline Imagenología & 2,8 & 3 \\
\hline Habilitación/rehabilitación & 2,9 & 3 \\
\hline Apoyo & 2,4 & 2 \\
\hline Direccionamiento & 2,3 & 2 \\
\hline Gerencia & 3,1 & 3 \\
\hline Gerencia del recurso humano & 2,4 & 2 \\
\hline Gerencia del ambiente físico & 2,3 & 2 \\
\hline Gerencia de la información & 2,2 & 2 \\
\hline Gestión de la tecnología & N/A & N/A \\
\hline Mejoramiento de la calidad & 2,3 & \\
\hline Sedes integradas en red & 2,9 & \\
\hline Mínimo & & \\
\hline Máximo & & \\
\hline
\end{tabular}

Fuente: Los autores, 2012.

En relación con la comparación de los dos modelos de autoevaluación, se encuentran congruencias y divergencias importantes en estructura, las cuales se plantean en el siguiente cuadro comparativo. 
Yolvi Amileth Rodríguez Navas, Juan Pablo Robayo Piñeros

Tabla 3. Comparación entre autoevaluación 9004:2009 y autoevaluación SUA

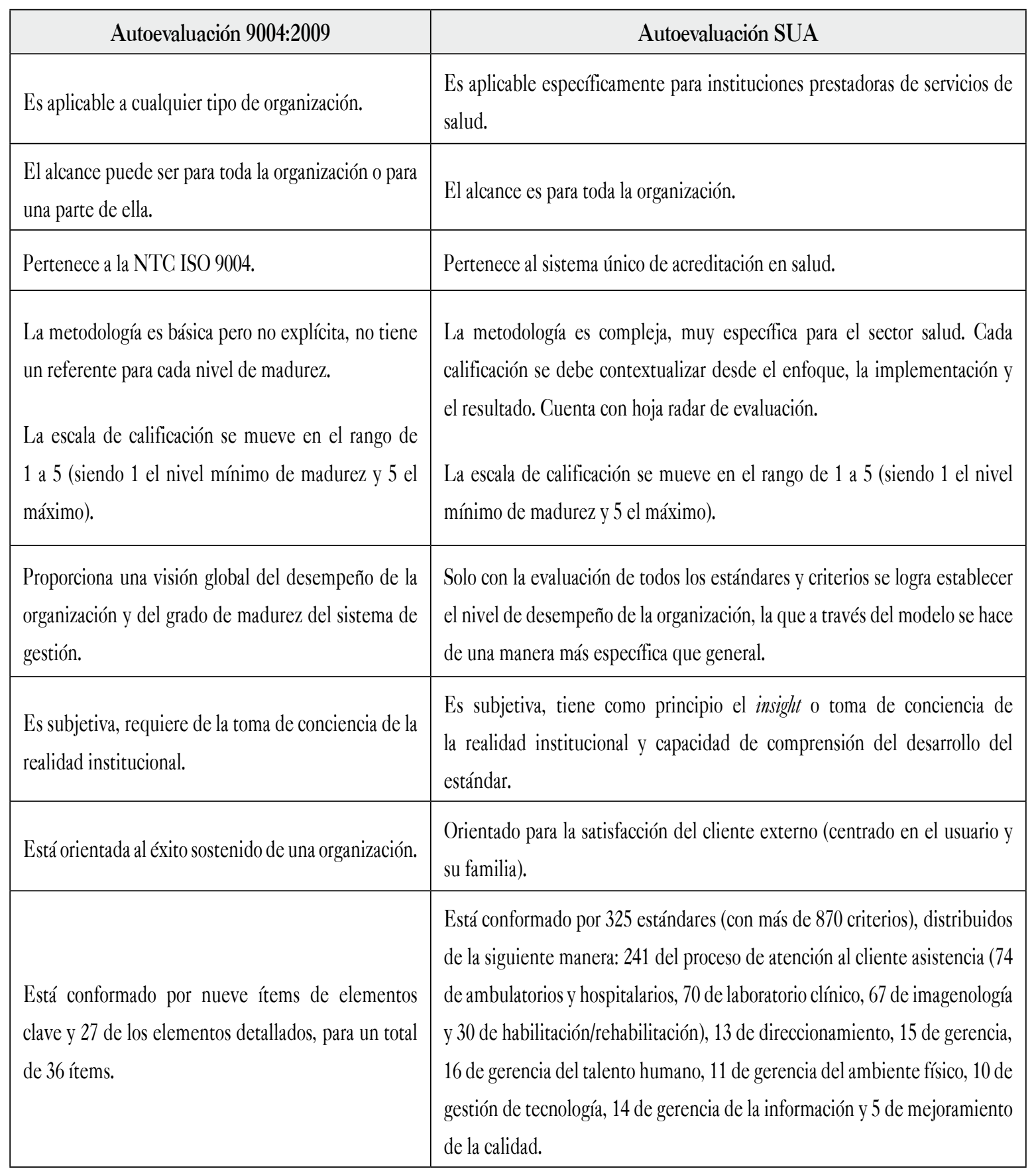


Ahora bien, al hacer la correspondencia de los elementos del modelo de autoevaluación de la ISO 9004:2009 y el modelo de autoevaluación del sistema único de acreditación (SUA) se encuentra lo siguiente:

\section{Tabla 4. Correspondencia entre elementos del modelo de autoevaluación ISO 9004:2009 y modelo de autoevaluación SUA}

\begin{tabular}{|c|c|}
\hline Elementos autoevaluación ISO 9004:2009 & Estándares autoevaluación sistema único de acreditación \\
\hline $\begin{array}{l}\text { Gestión } \\
\text { Gestión para el éxito sostenido basado en los ocho principios } \\
\text { de la calidad (enfoque al cliente, liderazgo, participación de las } \\
\text { personas, enfoque basado en procesos, enfoque de sistema para } \\
\text { la gestión, mejora continua, enfoque en hechos basados para la } \\
\text { toma de decisiones y relaciones mutuamente beneficiosas con } \\
\text { el proveedor). Incluye el entorno de la organización y las partes } \\
\text { interesadas. }\end{array}$ & $\begin{array}{l}\text { Gerencia } \\
\text { En forma general, establece el trabajo de las unidades funcionales } \\
\text { y de gobierno de la organización frente a las funciones clave que } \\
\text { debe desarrollar la institución. } \\
\text { En forma específica, define el sistema de gestión del riesgo } \\
\text { articulado con el direccionamiento estratégico; establece política } \\
\text { para la prestación de los servicios; determina procesos para } \\
\text { garantizar los recursos, las metas y objetivos; consecución de } \\
\text { la política organizacional; implementación de la política para la } \\
\text { humanización en la prestación de servicios. }\end{array}$ \\
\hline $\begin{array}{l}\text { Estrategia y política } \\
\text { La estrategia y la política son establecidas por la alta dirección, } \\
\text { la cual debe asegurarse de desplegarlas a toda la organización y } \\
\text { comunicarlas de manera eficaz, porque es un aspecto esencial } \\
\text { para el éxito sostenido. }\end{array}$ & $\begin{array}{l}\text { Direccionamiento } \\
\text { En forma general, establece el trabajo de la organización frente } \\
\text { a la planeación estratégica y el papel de los órganos de gobierno } \\
\text { de la institución. } \\
\text { En forma específica, construcción de plan estratégico, } \\
\text { despliegue del direccionamiento estratégico, diseño de política } \\
\text { de prestación de servicios, educación de directivas. Promoción } \\
\text { de la atención centrada en el usuario, el mejoramiento, la } \\
\text { humanización, la gestión del riesgo, la trasformación cultural y } \\
\text { la responsabilidad social. }\end{array}$ \\
\hline
\end{tabular}




\begin{tabular}{|c|c|}
\hline Elementos autoevaluación ISO 9004:2009 & Estándares autoevaluación sistema único de acreditación \\
\hline \multirow{4}{*}{$\begin{array}{l}\text { Recursos } \\
\text { La gestión de los recursos identifica los internos y los externos } \\
\text { necesarios para alcanzar los objetivos a corto y a largo plazo e } \\
\text { incluye: las necesidades financieras de la organización para } \\
\text { cubrir operaciones actuales y futuras; la gestión de las personas } \\
\text { como recurso significativo de la organización; los proveedores y } \\
\text { aliados por su interdependencia y las relaciones mutuamente } \\
\text { beneficiosas; la infraestructura gestionada de una manera } \\
\text { eficaz y eficiente; el ambiente de trabajo adecuado para lograr } \\
\text { y mantener el éxito sostenido; el conocimiento, la información } \\
\text { y la tecnología como recursos esenciales, y los recursos naturales } \\
\text { integrando aspectos de protección ambiental en los procesos y } \\
\text { productos / servicios. }\end{array}$} & $\begin{array}{l}\text { Gerencia del recurso humano } \\
\text { En forma general, se orienta a la gestión del talento humano en } \\
\text { toda su planeación (ingreso, retiro, mejora continua). } \\
\text { En forma específica, identificación de las necesidades del } \\
\text { talento humano, procesos para garantizar competencias, } \\
\text { educación, satisfacción, comunicación efectiva, trasformación } \\
\text { cultural, calidad de vida. }\end{array}$ \\
\hline & $\begin{array}{l}\text { Gestión de la tecnología } \\
\text { En forma general, se dirige a la gestión integral de los recursos } \\
\text { tecnológicos (planeación, renovación y análisis de los efectos de } \\
\text { su utilización. } \\
\text { En forma específica, establecimiento de procesos de la gestión } \\
\text { integral de la tecnología, diseño de política de gestión de } \\
\text { la tecnología (adquisición, seguridad del uso, renovación), } \\
\text { unificación de tecnologías, uso de equipos de última tecnología. }\end{array}$ \\
\hline & $\begin{array}{l}\text { Gerencia del ambiente físico } \\
\text { En forma general, establece los procesos que debe tener la } \\
\text { organización para que la estructura acompañe la adecuada } \\
\text { operación de los procesos asistenciales. }\end{array}$ \\
\hline & $\begin{array}{l}\text { En forma específica, identificación y cubrimiento de } \\
\text { necesidades del ambiente físico, seguridad del mismo, respuesta } \\
\text { ante emergencias, políticas de ambiente sano (no fumadores), } \\
\text { privacidad y confort del ambiente físico, gestión de residuos, } \\
\text { aplicación de nuevos diseños y tecnologías, seguridad del } \\
\text { ambiente. }\end{array}$ \\
\hline
\end{tabular}




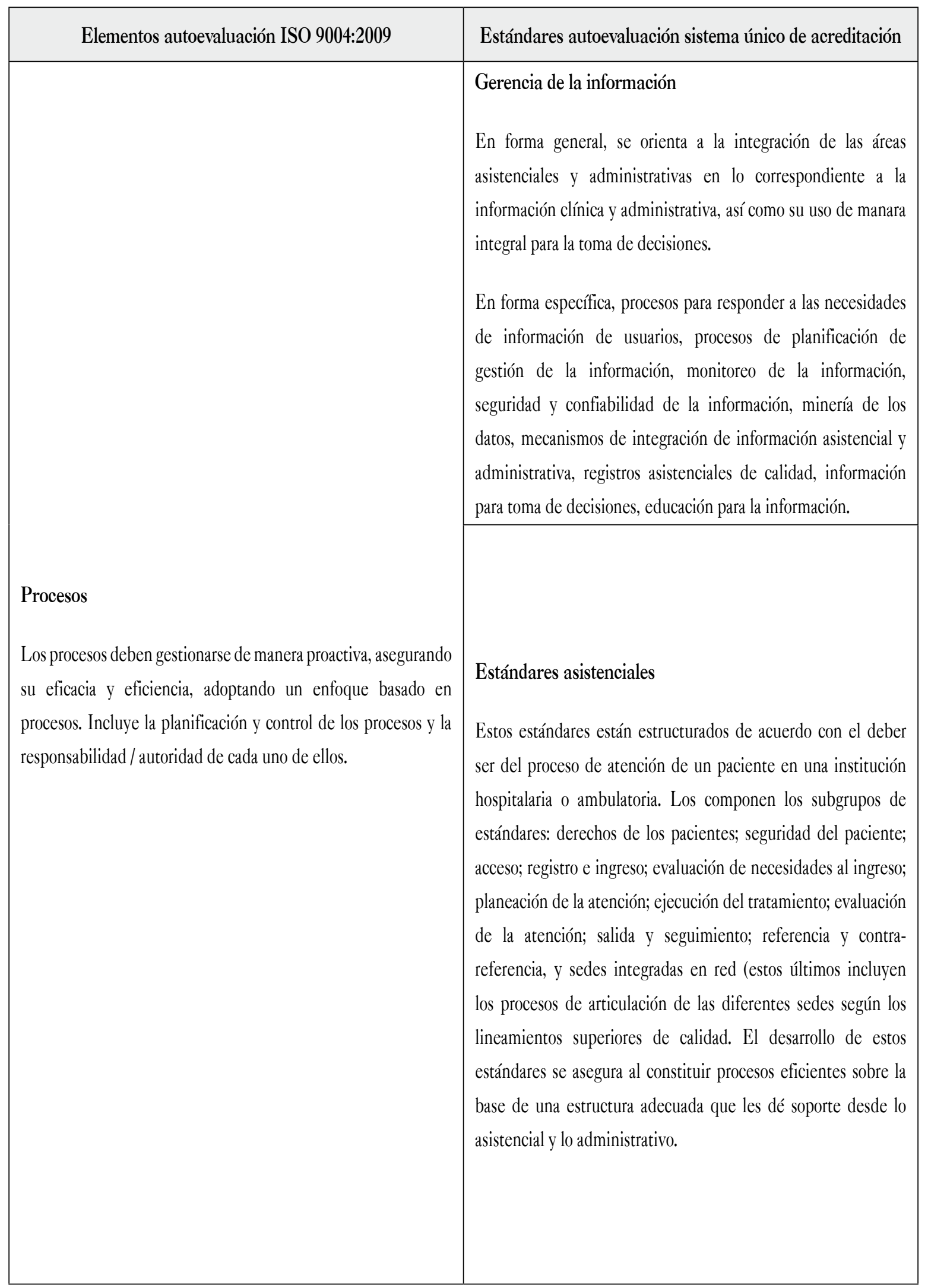




\begin{tabular}{|c|c|}
\hline Elementos autoevaluación ISO 9004:2009 & Estándares autoevaluación sistema único de acreditación \\
\hline $\begin{array}{l}\text { Seguimiento y medición } \\
\text { Seguimiento y medición para lograr el éxito sostenido ante } \\
\text { un entorno cambiante e incierto; incluye el seguimiento, la } \\
\text { medición, el análisis y la revisión de la información obtenida } \\
\text { para facilitar la toma de decisiones. }\end{array}$ & $\begin{array}{l}\text { El sistema único de acreditación (SUA) soporta la medición y } \\
\text { seguimiento de la gestión organizacional en una evaluación } \\
\text { permanente, orientada al logro de resultados centrados en el } \\
\text { usuario; evaluación de elementos medibles objetivamente; la } \\
\text { institución es la que establece la estrategia o mecanismo de } \\
\text { desarrollo de los estándares; uso de la metodología del paciente } \\
\text { trazador como un todo. }\end{array}$ \\
\hline $\begin{array}{l}\text { Mejora, innovación y aprendizaje } \\
\text { La mejora, innovación y aprendizaje como elementos necesarios } \\
\text { para el éxito sostenido. La mejora continua dentro del ciclo } \\
\text { PHVA; la innovación para satisfacer las necesidades y expectativas } \\
\text { de las partes interesadas, y el aprendizaje como organización y } \\
\text { como integrador de las capacidades de los individuos, lo que } \\
\text { permite aumentar la capacidad de la organización para gestionar } \\
\text { y mantener el éxito sostenido. }\end{array}$ & $\begin{array}{l}\text { Mejora de la calidad } \\
\text { El modelo posee cinco estándares de mejoramiento de la } \\
\text { calidad que aplican a todos los procesos evaluados (asistenciales } \\
\text { y de apoyo). } \\
\text { Además, cada grupo de estándares cuenta con un estándar } \\
\text { de mejoramiento de la calidad que permite evaluar cómo los } \\
\text { requisitos de los estándares del grupo de mejoramiento se } \\
\text { utilizan en la revisión del grupo específico. } \\
\text { El componente innovación no está contemplado en forma } \\
\text { explícita como elemento clave de gestión. } \\
\text { El componente aprendizaje organizacional está inmerso en } \\
\text { diferentes estándares, en algunos casos de manera explícita y en } \\
\text { otros de manera tácita. }\end{array}$ \\
\hline
\end{tabular}

Fuente: Los autores, 2012.

Se logró estructurar un modelo integrador que permite conocer el nivel de madurez de la gestión organizacional de una institución prestadora de servicios de salud, teniendo en cuenta dentro de un contexto integrador los elementos del modelo de autoevaluación de la NTC ISO 9004:2009 así como los elementos específicos del sector salud abordados en el modelo de autoevaluación del sistema único de acreditación (SUA).
El instrumento se divide en dos partes, una que evalúa los elementos clave, tales como: gestión, estrategia y política, recursos, procesos, seguimiento y medición, mejora, innovación y aprendizaje; y la otra evalúa elementos detallados donde se incluyen, además de las áreas contempladas en la primera parte, los procesos de atención al cliente asistencial. 


\section{Tabla 5. Elementos clave}

\begin{tabular}{|l|l|}
\hline No. & Elementos clave \\
\hline 1 & ¿Cuál es el centro de interés de la dirección? \\
\hline 2 & ¿Cuál es el enfoque del liderazgo? \\
\hline 3 & ¿Cómo obtiene mejores resultados la dirección? \\
\hline 4 & ¿Cómo se decide qué es importante? \\
\hline 5 & ¿Qué se necesita para obtener resultados? \\
\hline 6 & ¿Cómo se organizan las actividades? \\
\hline 7 & ¿Cómo se logran los resultados? \\
\hline 8 & ¿Cómo se realiza el seguimiento de los resultados? \\
\hline 9 & ¿Cómo se deciden las prioridades de mejora? \\
\hline 10 & ¿Cómo tiene lugar el aprendizaje? \\
\hline 11 & ¿Para quién es útil el aprendizaje? \\
\hline
\end{tabular}

Tabla 6. Elementos detallados

\begin{tabular}{|l|l|}
\hline No. & Elementos detallados \\
\hline 1 & Generalidades \\
\hline 2 & Éxito sostenido \\
\hline 3 & El entorno de la organización \\
\hline 4 & Partes interesadas, necesidades y expectativas \\
\hline 5 & Generalidades \\
\hline 6 & Formulación de la estrategia y la polííca \\
\hline 7 & Despliegue de la estrategia y la políica \\
\hline 8 & Comunicación de la estrategia y la política \\
\hline 9 & Generalidades \\
\hline 10 & Recursos financieros \\
\hline 11 & Talento humano - Generalidades \\
\hline 12 & Talento humano - Aplicación de procesos \\
\hline 13 & Talento humano - Competencias \\
\hline 14 & Proveedores y aliados \\
\hline 15 & Infraestructura \\
\hline 16 & Ambiente físico \\
\hline 17 & Conocimientos, información y tecnología - Generalidades \\
\hline 18 & Gestión de tecnología \\
\hline 19 & Gerencia de la información \\
\hline 20 & Recursos naturales \\
\hline 21 & Gestión ambiental \\
\hline 22 & Planificación y control de los procesos \\
\hline
\end{tabular}




\begin{tabular}{|l|l|}
\hline No. & Elementos detallados \\
\hline 23 & Responsabilidad y autoridad relativas a los procesos \\
\hline 24 & Derechos de los pacientes \\
\hline 25 & Seguridad del paciente \\
\hline 26 & Acceso \\
\hline 27 & Registro - Ingreso \\
\hline 28 & Necesidades de ingreso \\
\hline 29 & Planeación de la atención \\
\hline 30 & Seguimiento a QRS \\
\hline 31 & Ejecución del tratamiento \\
\hline 32 & Evaluación de la atención \\
\hline 33 & Proceso de planeación \\
\hline 34 & Salida y seguimiento \\
\hline 35 & Referencia / contrarreferencia \\
\hline 36 & Sedes integradas \\
\hline 37 & Espera previa a la atención en el servicio de laboratorio clínico \\
\hline 38 & Control de calidad en el servicio de laboratorio clínico \\
\hline 39 & Entrega de resultados en el servicio de laboratorio clínico y en servicio de imagenología \\
\hline 40 & Remisión a urgencias / hospitalización desde el servicio de imagenología \\
\hline 41 & Seguimiento \\
\hline 42 & Medición: generalidades e indicadores clave de desempeño \\
\hline 43 & Medición: auditoría interna y autoevaluación \\
\hline 44 & Estudios comparativos con las mejores prácticas (benchmarking) \\
\hline 45 & Análisis \\
\hline 46 & Revisión de la información obtenida del seguimiento, la medición y el análisis \\
\hline 47 & Mejora continua \\
\hline 48 & Innovación \\
\hline 49 & Aprendizaje organizacional \\
\hline
\end{tabular}

Fuente: Los autores, 2012.

Para realizar la evaluación de los elementos de esta herramienta, primero que todo debe definirse el responsable del proceso y cuándo se llevará a cabo. Es importante tener en cuenta que para la evaluación de los elementos clave el responsable debe ser miembro de la alta dirección o un delegado, mientras que los elementos detallados pueden ser evaluados por los miembros de la oficina de aseguramiento de la calidad o los líderes de los procesos.
La matriz debe diligenciarse marcando con una $\mathrm{X}$ el nivel que en la descripción esté más cerca a la realidad de la institución, lo que se verifica con documentos existentes. Este nivel reporta al mismo tiempo un puntaje que se tiene en cuenta para obtener los resultados. Ejemplo: el nivel 1 equivale a una puntuación de 1 y el 5 , a una de 5 . 
Los resultados pueden obtenerse de dos maneras: la primera es hallar la media y la moda de los puntajes obtenidos, y la segunda es sumar los puntajes marcados y clasificarlos en los siguientes rangos:

Tabla 7. Rangos de clasificación

\begin{tabular}{|c|c|}
\hline \multicolumn{2}{|c|}{ Elementos clave } \\
\hline Rango & Nivel \\
\hline 11 a 19 & 1 \\
\hline $20 \mathrm{a} 28$ & 2 \\
\hline 29 a 37 & 3 \\
\hline 38 a 46 & 4 \\
\hline 47 a 55 & 5 \\
\hline
\end{tabular}

\begin{tabular}{|c|c|}
\hline \multicolumn{2}{|c|}{ Elementos detallados } \\
\hline Rango & Nivel \\
\hline 49 a 88 & 1 \\
\hline 89 a 127 & 2 \\
\hline 128 a 166 & 3 \\
\hline 167 a 205 & 4 \\
\hline 206 a 245 & 5 \\
\hline
\end{tabular}

\begin{tabular}{|c|c|}
\hline \multicolumn{2}{|c|}{ Puntaje total } \\
\hline Rango & Nivel \\
\hline 60 a 107 & 1 \\
\hline 108 a 155 & 2 \\
\hline 156 a 203 & 3 \\
\hline 204 a 251 & 4 \\
\hline 252 a 300 & 5 \\
\hline
\end{tabular}

Fuente: Los autores, 2012.

Dentro de los resultados se pueden encontrar áreas de mayor nivel de madurez que otras, lo cual puede identificarse mediante la gráfica radar. De esta manera se centran los planes de mejora en áreas que necesitan pasar a un nivel superior.

Ahora, para definir que el nivel de madurez de la gestión organizacional de la IPS objeto de estudio con el modelo integrado de la NTC ISO 9004:2009 y el sistema único de acreditación (SUA) es de 3, se toma como resultado el valor obtenido de la media y la moda.

Adicionalmente y teniendo en cuenta los rangos propuestos para medir el nivel de madurez organizacional, los resultados son: el puntaje obtenido en la evaluación de los elementos clave fue de 32, lo que corresponde a nivel 3 de madurez organizacional; el puntaje obtenido en elementos detallados es de 143 , correspondiendo al nivel 3 de madurez organizacional, y finalmente el puntaje total obtenido es de 175 , que indica que esta institución tiene un nivel de madurez de 3, lo que significa que su sistema es definido, es decir que los procesos están estandarizados y se ejecutan de acuerdo con lo documentado; ello es la base de su mejoramiento. Existe un entendimiento de las relaciones mutuas entre actividades y medidas del proceso.
Tabla 8. Evaluación de elementos clave y detallados del modelo integrado

\begin{tabular}{|c|c|}
\hline \multicolumn{2}{|l|}{ Elementos clave } \\
\hline Área & Promedio \\
\hline Gestión & 2,6 \\
\hline Estrategia y política & 3 \\
\hline Recursos & 4 \\
\hline Procesos & 3 \\
\hline Seguimiento y medición & 2,5 \\
\hline Mejora, innovación y aprendizaje & 3 \\
\hline Total & 3,0 \\
\hline \multicolumn{2}{|l|}{ Elementos detallados } \\
\hline Área & Promedio \\
\hline Gestión para el éxito sostenido & 3,25 \\
\hline Estrategia y política & 3,25 \\
\hline Gestión de recursos & 2,69 \\
\hline Procesos & 2,5 \\
\hline Procesos de atención al cliente asistencial & 2,81 \\
\hline Seguimiento, medición y análisis & 3,33 \\
\hline Mejora, innovación y aprendizaje & 3,33 \\
\hline Total & 3,0 \\
\hline
\end{tabular}

Nivel de madurez $=3$

Fuente: Los autores. 
$\mathrm{Al}$ comparar los resultados finales de cada modelo se define que el nivel de madurez de la gestión organizacional del hospital evaluado es de 3, con una media de 2,8 y una moda de 3.

\section{CONCLUSIONES}

El nivel de madurez de la IPS objeto de estudio arrojó resultados diferentes con la autoevaluación por medio del modelo ISO 9004:2008 vs. el sistema único de acreditación (SUA), encontrándose un valor de 3 (con un mínimo de 2 y un máximo de 4 en los valores de los elementos clave y los elementos detallados) para el primero y un nivel de madurez de 2,6 de promedio y de 2 obtenido de la moda (téngase en cuenta que el sistema recomienda calificar usando como base el menor valor obtenido en la autoevaluación de los criterios), con puntajes que fluctúan con un valor mínimo de 2,3 y uno máximo de 2,9 para el segundo.

La correlación de los dos modelos de autoevaluación permite encontrar congruencias importantes y elementos afines que pueden incorporarse a la propuesta de un nuevo modelo. Dentro de los elementos afines y compatibles están los componentes del direccionamiento y la gerencia, los que si bien es cierto están incluidos en los dos modelos, en la autoevaluación de la ISO 9004:2009 presentan un mayor desarrollo y profundidad dentro de un enfoque claro al logro del éxito sostenido, no así su abordaje en el SUA, modelo en el que su desarrollo es de menor grado sin una visión explícita hacia este fin. De igual manera, elementos como procesos y recursos están presentes en los dos modelos, con la salvedad de la especificidad de cada uno de ellos para el sector hospitalario en el SUA.

Asimismo, las mayores divergencias de los dos modelos, además de la alta especificidad para el sector salud del SUA y que como bien se sabe no lo tiene la ISO 9004, se centran básicamente en la carencia o por lo menos lo poco explícito del SUA en los siguientes aspectos: un direccionamiento con enfoque en el éxito sostenido, como se mencionó anteriormente, unos lineamientos claros de innovación y aprendizaje organizacional, y el pobre interés por el ambiente externo que rodea la organización. Estos propósitos están muy claros y bien estructurados en el modelo ISO 9004.

El proceso de autoevaluación en el SUA se hace frente a criterios definidos para la prestación de servicios de salud, lo cual lo vuelve muy específico y sectorial al estructurarse sobre estándares misionales del sector sanitario. Sin embargo, el ejercicio de su aplicación resulta extenso, complejo, poco comprensible frente al qué del estándar, situación que desmotiva a las IPS interesadas en lograr la acreditación en salud. Prueba de ello es el número tan reducido de centros hospitalarios acreditados en Colombia, con tan solo 25 instituciones en todo el país.

El modelo integrado que se propone en este estudio articula requisitos de los dos modelos dentro de la orientación de coherencia y complementariedad de ambos, lo cual lo hace un instrumento que facilita el proceso de autoevaluación del nivel de madurez de organizaciones como las instituciones prestadoras de servicios de salud, con la ventaja de que, al tomar elementos tanto de la ISO 9004 como del SUA, se estructura una herramienta con criterios específicos para el sector salud con un enfoque de direccionamiento para el éxito sostenido.

Este modelo fue validado en contenido por expertos y de acuerdo con el índice de congruencia de Osterlind que obtuvo cada uno de los ítems del instrumento. Son congruentes con el objetivo de la herramienta, la cual contiene 60 ítems, 11 de evaluación de elementos clave y 49 de elementos detallados, donde se mantiene la estructura general del instrumento de autoevaluación de la ISO 9004:2008, pero se introducen 25 
ítems específicos del sector salud, propios del SUA, reduciendo los 158 estándares (aproximadamente 500 criterios) del modelo de acreditación en salud.

\section{RECOMENDACIONES}

Es necesario validar el instrumento propuesto con métodos estadísticos que garanticen la confiabilidad de este y la aplicación a una muestra significativa de instituciones prestadoras de servicios de salud de alto nivel de complejidad, y luego entregarlo a la oficina de ICONTEC responsable del área de acreditación en salud para que estudien la posibilidad de incorporarlo en el modelo del SUA.

Se sugiere proponer un macroproyecto de calidad en salud que incentive a estudiantes de la Maestría en Calidad y Gestión Integral y que tengan como base profesiones de salud, para que desarrollen investigaciones, profundicen y generen conocimiento en el tema.

Se recomienda generar proyectos que promuevan la integración del sistema de acreditación en salud y la ISO 9004.

\section{BIBLIOGRAFÍA}

COLOMBIA. MINISTERIO DE SALUD Y PROTECGIÓN SOCIAL. Guía práctica del sistema obligatorio de garantía de la calidad en salud. Bogotá: Ministerio de Salud y Protección Social, marzo, 2011. $98 \mathrm{p}$.

_- Decreto 1011: Por el cual se establece el sistema obligatorio de garantía de la calidad de la atención de salud del sistema general de seguridad social en salud (SGSSS). Bogotá: Ministerio de Salud y Protección Social, 3, abril, 2006. 17 p.

Guía práctica de la preparación para la acreditación en salud. Bogotá: Editorial
Imprenta Nacional de Colombia, 2007. 101 p. ISBN: 978-958-98220-8-1.

Pautas de auditoría para el mejoramiento de la calidad de la atención en salud. Bogotá: Editorial Imprenta Nacional de Colombia, 2007. 72 p. ISBN: 978-958-98220-6-7.

Prestación de servicios de salud. 2012. [consultado 5 de julio, 2012]. Disponible en http://201.234.78.38/SIHO/informes/ipspornivel. aspx? pageTitle=IPS\%20por\%20Nivel\%20de\%20 Atenci\%F3n\&pageHlp=/SIHO/ayudas/informes/ caracterizacion.pdf

Resolución 1446: Por la cual se define el sistema de información para la calidad y se adoptan los indicadores de monitoría del sistema obligatorio de garantía de calidad de la atención en salud. Anexo Técnico. Bogotá: Ministerio de Salud y Protección Social, 8 de mayo, 2006. 83 p.

—_ Resolución 1445: Por la cual se definen las funciones de la entidad acreditadora y se adoptan otras disposiciones. Anexo Técnico 2. Bogotá: Ministerio de Salud y Protección Social, 2006. 14 p.

— Resolución 123: Por la cual se modifica el artículo 2 de la Resolución 1445 de 2006. Anexo Técnico. Bogotá: Ministerio de Salud y Protección Social, 2012.84 p.

COLOMBIA. SECRETARÍA DISTRITAL DE SALUD DE BOGOTÁ. Instructivo para el diligenciamiento de la autoevaluación para la habilitación y novedades de apertura de profesionales independientes e IPS. Anexo Técnico No. 5. Bogotá: Secretaría Distrital de Salud de Bogotá. 2009. 9 p.

ESPINOSA, Fernando F.; DÍAZ, Acires y BACK, Nelson. Un procedimiento de evaluación de las condiciones necesarias para innovar la 
gestión de mantenimiento en una empresa. [consultado 16 de noviembre, 2011]. Disponible en http://web.ebscohost.com/ehost/detail? vid=3\&hid=104\&sid=477761b1-7593-41ad-b8d c00e5b4ef682f\%40sessionmgr112\&bdata=Jmxh bmc9ZXMmc210ZT1laG9zdC1saXZl\#db=a9h\& $\mathrm{AN}=31464154$

INSTITUTO COLOMBIANO DE NORMAS TÉCNICAS Y CERTIFICACIÓN. Normas fundamentales sobre gestión de la calidad y documentos de orientación para su aplicación. Gestión para el éxito sostenido de una organización. Enfoque de Gestión de la Calidad NTC - ISO 9004:2009. 3 ed. Bogotá, 2010.
Acreditación en salud. 2012. [consultado 5 de julio, 2012]. Disponible en http://www.acreditacionensalud.org.co/catalogo/docs/IPSacreditadas.pdf

MORALES, Pedro. Las pruebas objetivas. España: Editorial Universidad de Deusto, 2009. 60 p.

ORGANISMO NACIONAL DE ACREDITACIÓN DE COLOMBIA. La acreditación en Colombia. Bogotá, 2010.32 p.

TAMAYO, Mario. El proceso de la investigación científica. México: Editorial Limusa, 2003. 46 p. 